\title{
Clinical characteristics of head and neck cancers at a tertiary care hospital
}

\author{
Sajid Durrani ${ }^{1}$, Saleh Al-Dhahari ${ }^{2}$, Haneen Sebeih ${ }^{2}$, Tariq Wani ${ }^{3}$, Khalid Al Qahatani ${ }^{4}$, Humariya Heena ${ }^{3}$
}

\author{
AFFILIATION \\ 1 Comprehensive Cancer Center, King Fahad Medical City, Riyadh, Saudi \\ Arabia \\ 2 Department of Otolaryngology, Head and Neck Surgery, King Fahad \\ Medical City, Riyadh, Saudi Arabia \\ 3 King Fahad Medical City Research Center, Riyadh, Saudi Arabia \\ 4. Department of Head and Neck Surgery, King Saud University, Riyadh, \\ Saudi Arabia
}

CORRESPONDENCE TO

Sajid Durrani. Comprehensive Cancer Center, King Fahad Medical City, Riyadh, Saudi Arabia. E-mail: sdurrani@kfmc.med.sa

\section{KEYWORDS}

head and neck cancer, oral cavity cancer, squamous cell carcinoma, tobacco diseases, treatment failures, metastasis

Received: 10 January 2020, Accepted: 4 April 2020

https://doi.org/10.18332/popmed/120072 including oral cavity, nasopharynx, oropharynx, pharynx, larynx, sinonasal, and salivary glands were included. The endpoints were to assess tumour site of HNC, frequency of HNC based on histology, tumor-node-metastasis (TNM) staging, and etiological factors of HNC. The pattern of treatment failure was also recorded in reference to the site of tumour recurrence as local, general or distant.

RESULTS Out of 1349 patients screened, 652 were confirmed with HNC. The most common primary tumor site was the oral cavity (25.9\%), followed by nasopharynx (23.8\%), and larynx (17.5\%). Squamous cell carcinoma was the most common HNC (78.1\%). Based on TNM staging, the frequency of HNC varied from stage 0 to 4 , with $64.6 \%$ of patients in stage 4 . Infection with Epstein-Barr virus (EBV) was found in $8.5 \%$ of patients. Treatment failures were recorded in $24.3 \%$ of patients, and $62.5 \%$ of patients reported metastasis.

CONCLUSIONS High tobacco consumption in the region needs to be monitored and controlled by awareness and education campaigns. Effective management of HNC with early diagnosis and treatment is required by healthcare professionals to improve the overall quality of life of HNC patients.

\section{INTRODUCTION}

Head and neck cancer (HNC) is considered as the ninth most common cancer worldwide. According to the World Health Organization (WHO), more than 0.55 million annual cases of HNC occur worldwide, with around 0.30 million deaths each year ${ }^{1}$. In Saudi Arabia also, the incidence of HNC is high. According to the cancer data factsheet of Saudi Arabia, there were 769 cases registered in the HNC category in 2018, which resulted in 378 deaths.

Consumption of tobacco/smokeless tobacco/alcohol, infection with Epstein-Barr virus (EBV) and human papillomavirus (HPV) are considered the main etiological factors leading to HNC. These factors have recently been on the rise especially in the higher age group (40-55 years) in 
the last few years around the world including Saudi Arabia ${ }^{2-4}$, leading to metastatic or locoregional or viral-associated HNC.

Different malignancies that develop in or around the throat, larynx, nasopharynx, nose, sinuses and mouth are categorized under $\mathrm{HNC}^{5}$. Out of various histological types in $\mathrm{HNC}$, such as blastomas, lymphomas, sarcomas and neuroendocrine tumors, the majority is of epithelial origin (squamous cell carcinoma) and accounts for more than $90 \%$ of $\mathrm{HNCs}^{2}$. Nasopharyngeal cancer (NPC) is one of the rare forms of HNC. It has been ranked first among all HNCs in Saudi Arabia, with male predominance (male: female $=2: 1)^{6,7}$.

Cancer of head and neck is considered difficult to understand beyond a very early stage and shows a poor survival rate of five years ${ }^{8}$. Moreover, the published incidence of distant metastases (DM) in HNC treated for squamous cell carcinoma varies between $4 \%$ and $26 \%{ }^{9}$. Nevertheless, the overall rate has been improved from $52.7 \%$ in the period 1982-1986 to $65.9 \%$ during 2002-2006, which might be due to the use of advanced treatment modalities in $\mathrm{HNC}^{10}$.

The HNCs are quite common in Saudi Arabia due to a very high incidence of smoking and other risk factors ${ }^{11}$. It is extremely critical to document the pattern of treatment failure, especially on the Saudi population. However, in an extensive literature search, we found that comprehensive studies documenting extensive variables related to HNC to be lacking in Saudi Arabia. Therefore, the present study aimed to understand the clinical characteristics in patients with HNC treated at King Fahad medical city (KFMC).

\section{METHODS}

\section{Study population}

All histopathologically confirmed patients with cancer at sites including the oral cavity, nasopharynx, oropharynx (tongue, floor of the mouth, retromolar trigone, gingiva, tonsil, and lip), pharynx, larynx, sinonasal and salivary glands were included in the study. Patients with cancer at other sites or with incomplete data were excluded from the study.

\section{Study design}

A hospital-based retrospective closed cohort study design was employed to assess the clinical characteristics in patients with advanced HNC treated at KFMC, which is a tertiary care center. The medical records of all the patients who were diagnosed with HNC (in the period 2009-2018) and treated at KFMC were assessed from the tumor boards at the centre and reviewed.

\section{Study endpoints}

The endpoints of the study were to assess the clinical characteristics (tumor site of HNC, frequency of HNC based on histology, TNM staging and etiological factors) of HNC in Saudi Arabia.

Tumor-node-metastasis (TNM) staging was followed to categorize the different stages of the disease. The categorization of stages T0 to T4 is based on the presence of a primary tumor and its size. A tumor is categorized as T0 if there is no evidence of primary tumor. The advanced stages (T1, T2, T3 and T4) are dependent on the size of the tumor and its extension. The TNM staging varies a little in the description as per the subtype of $\mathrm{HNC}$, but the basic categorization is similar for all types of $\mathrm{HNCs}^{12}$. The nodal status of the tumor is based on the involvement of lymph nodes. In N0 (nodal stage 0 ), no regional lymph node metastasis is involved, while in the next stages, there is metastasis at the lymph node and the size of tumor increases in more advanced stages ${ }^{12}$. The failure rates were recorded in reference to whether the recurrence was local, general or distant.

\section{Statistical analysis}

Data were analyzed descriptively and presented as frequency (n) and percentage (\%). The statistical package for social sciences (IBM SPSS statistics 22.Ink) was used for data analysis.

\section{RESULTS}

\section{Study population}

A total of 1349 patients were screened for HNC in KFMC in the period 2009-2018, of which 652 patients presented with HNC. Out of 652, 416 (63.8\%) were males and 236 (36.2\%) were females (Table 1). More than $80 \%(n=541 ; 82.9 \%)$ of the patients were of Saudi nationality while the rest $(n=111$; $17.1 \%$ ) were non-Saudi patients (Table 1). The new cases of HNC registered at KFMC were 376 (57.6\%) during the study, while 276 (42.4\%) had been reported previously (Table 1).

\section{Tumor site}

The oral cavity was found to be the most common primary tumor site in 169 (25.9\%) patients, closely followed by nasopharynx in 155 (23.8\%) patients. A total of 114 (17.5\%) patients were reported to have the larynx as their tumor site. The sinonasal, salivary glands, pharynx and oropharynx were also reported as tumor sites in 147 (22.6\%) patients (Figure 1).

Table 1. Sociodemographic characteristics of the head and neck cancer patients involved in the study, Saudia Arabia $(\mathrm{N}=652)$

\begin{tabular}{lcc} 
Characteristics & n & $\%$ \\
\hline Sex & & \\
Male & 416 & 63.8 \\
Female & 236 & 36.2 \\
Residence & & \\
Saudi Arabia & 541 & 82.9 \\
Other & 111 & 17.1 \\
\hline Registered cases & & \\
New & 376 & 57.6 \\
Previous & 276 & 42.4
\end{tabular}




\section{Frequency of HNC based on histology}

In regard to histology, squamous cell carcinoma was the most abundantly found form of HNC in 509 (78.1\%) patients, while $67(10.2 \%)$ patients had salivary gland cancers (Figure 2).

\section{Frequency of HNC based on TNM staging}

Of the 652 HNC patients, 285 (43.8\%) had stage T4 disease, while $117(18.0 \%)$ patients were at stage T3. A total of 91 (13.9\%), $111(17.0 \%)$ and $48(7.3 \%)$ patients were reported at stages $\mathrm{T} 2, \mathrm{~T} 1$ and $\mathrm{T} 0$, respectively. In nodal categorization, $306(47.0 \%)$ patients were in N0 stage and 203 (31.1\%) patients were in stage N2. In all, 9.9\% of patients reported no distant metastasis (M0 stage), while 421 (64.6\%) patients had stage-IV metastatic (M4) disease. Figure 3 represents the frequency of head and neck cancer based on TNM staging.

\section{Frequency of HNC based on etiological factors}

In the present study, EBV infection was positive in $8.5 \%$ of the study population.

\section{Patterns of failure in the treatment of HNC patients}

There were a total of $159(24.3 \%)$ treatment failures among

Figure 1. Different sites of the tumor among head and neck cancer patients, Saudi Arabia (N=652)

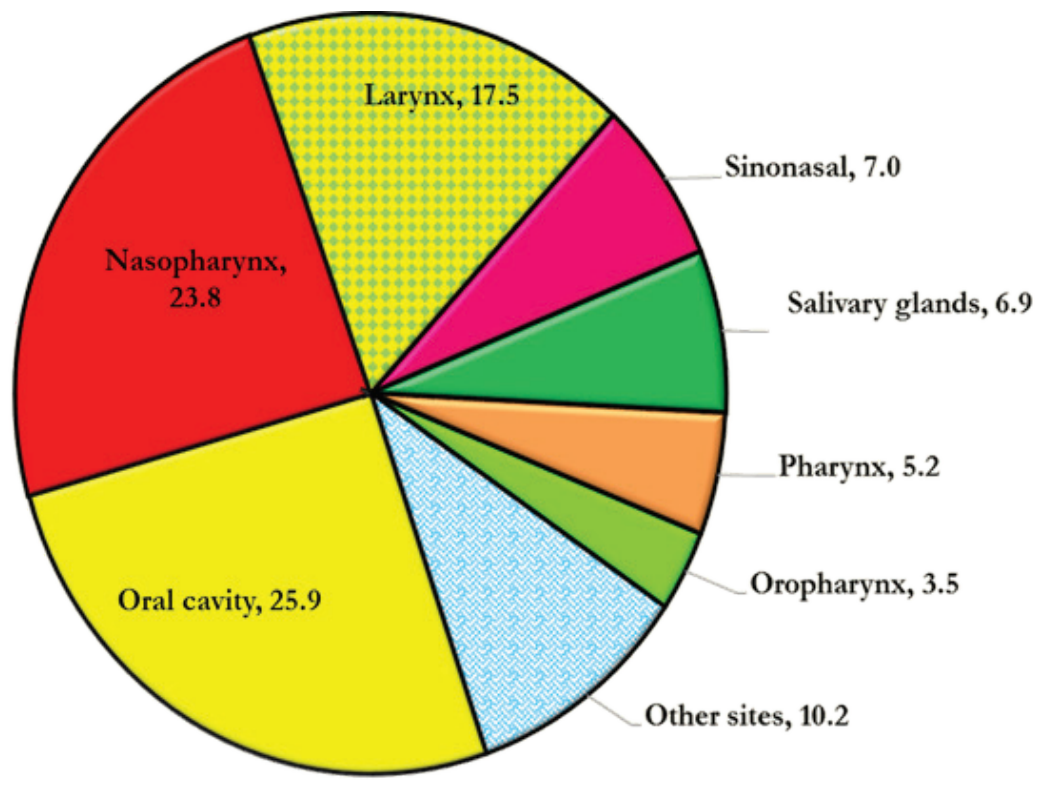

Figure 2. Histology of head and neck cancer among Saudi patients $(\mathrm{N}=652)$

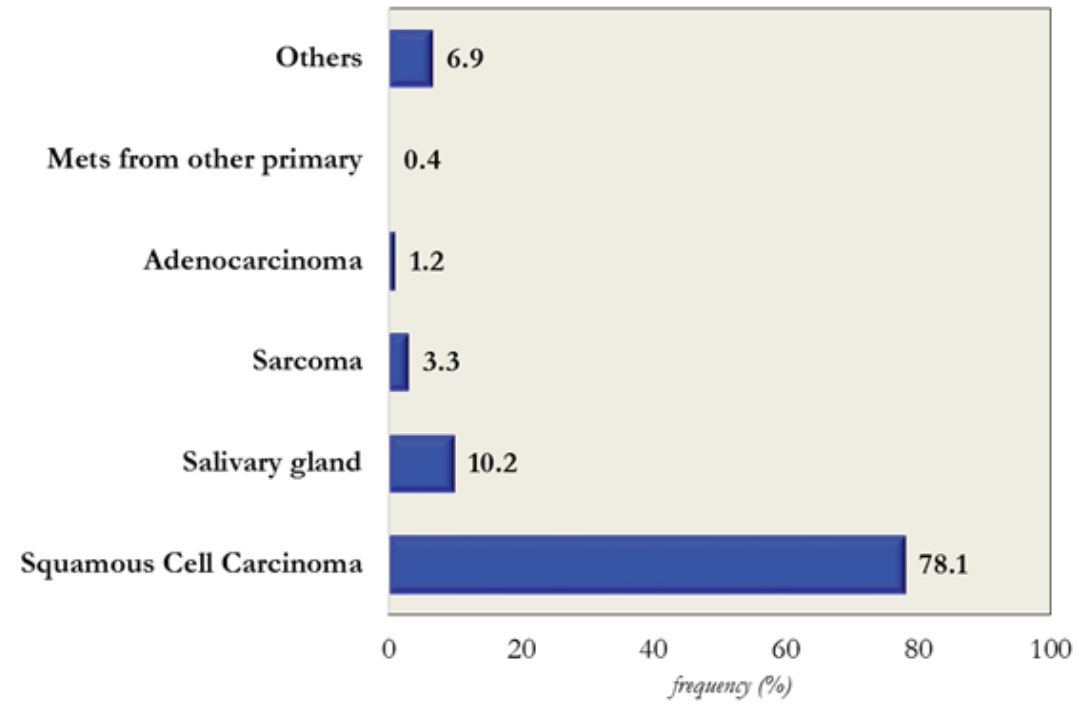




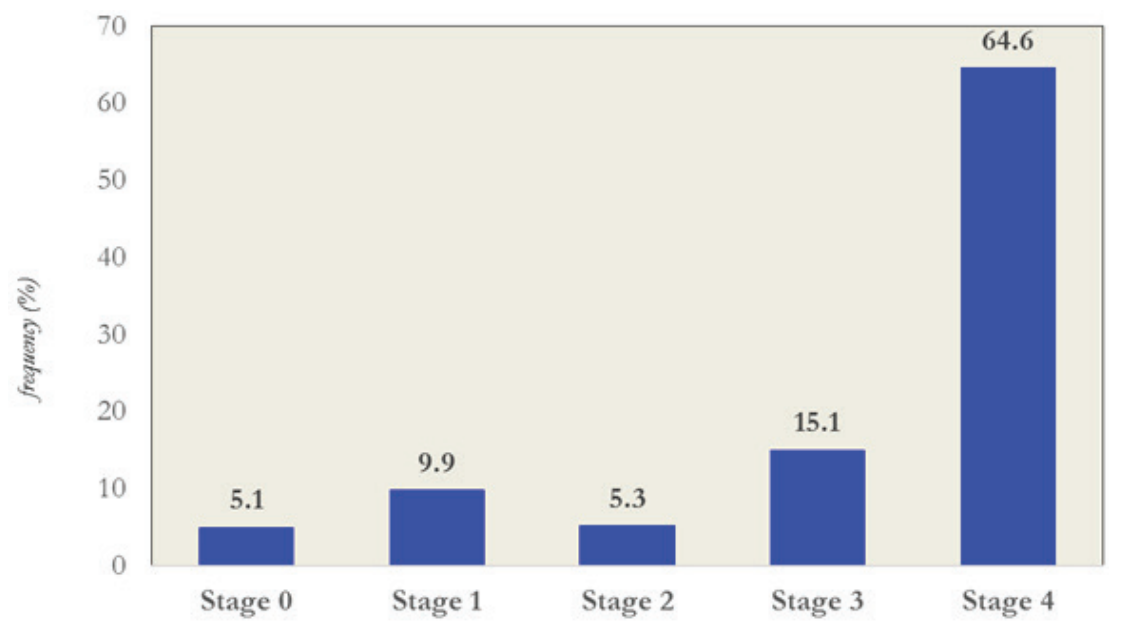

which, the local failures were more in laryngeal, followed by nasopharyngeal and oral cavity cancers. However, nasopharyngeal and oral cavity cancers had the highest number of regional failures as well as distant failures. Notably, 407 (62.5\%) patients were recorded for metastasis during their subsequent visits to the centre at KFMC.

\section{DISCUSSION}

Cancer of the head and neck is a heterogeneous disease with high incidence rates in the world. Only $3 \%$ of all malignant tumors are reported to be present in Europe and the United States ${ }^{5,8}$. However, it is considered as one of the common malignancies in South-Central and South-East Asia ${ }^{13}$, clearly depicting the role of geographic factors in HNC incidence. Therefore, it is important to understand the clinical characteristics and prognostic risk factors of patients in Saudi Arabia.

Cancer of the head and neck are highly prevalent in Saudi Arabia. In an interview conducted in a sample of 124 adult patients in a major tertiary hospital in Saudi Arabia, HNC was the second most common cancer affecting $15.3 \%$ of the study population, after breast cancer $(27.4 \%)^{14}$. In the present study as well, $48.33 \%$ had HNC, among which males were more commonly affected with HNC compared to females $(416 ; 63.8 \%$ vs $236 ; 36.2 \%)$. Similar results were described in other studies where the number of male patients with HNC was comparatively more than females ${ }^{6}$.

Tobacco chewing is one of the major risk factors behind oral cancers in Saudi Arabia ${ }^{5,7}$. According to a study conducted in Riyadh, Saudi Arabia, it was reported that a majority (58\%) of HNC patients were tobacco users, further leading to high incidence of oral cancer ${ }^{15}$. Our study also reported oral cancer as the most frequent site of HNC, affecting $25.9 \%$ of patients that might be due to high tobacco consumption.

Histologically, head and neck squamous cell carcinoma
(HNSCC) is the most frequent subtype of HNC worldwide ${ }^{16}$. In concordance, the present study also showed nearly $78.1 \%$ of cases of HNSCC. In recent times, the incidence of nasopharyngeal squamous cell carcinoma (NPSCC) has increased in Arab countries, including Saudi Arabia. A total of $23.8 \%$ of cases of nasopharyngeal cancer were also reported in our study too, which was higher in comparison to other subtypes of HNC. This increase can be attributed to the increased exposure to related risk factors such as tobacco smoking, alcohol consumption, preserved food intake, exposure to different types of medicines, and family history of NPC and EBV infection ${ }^{17-20}$.

Epstein-Barr virus (EBV) belongs to the family of the Herpes virus and has a ubiquitous presence with nearly $90 \%$ of the human adult population infected by the virus. Saliva is the main transmitter of the virus, causing HNC. EBV usually remains dormant without showing any symptoms, but sometimes, the virus can revert to its lytic state and transform the cells into malignant tumors ${ }^{17}$. Few studies are available regarding the relationship between EBV and HNC in Saudi Arabia. One study published in 1994 reported an examination of 25 Saudi patients with HNC (NPC) for the presence of EBV and revealed $92 \%$ of the tumor specimens positive for EBV DNA ${ }^{21}$. However, in the present study, EBV infection was positive in only $8.5 \%$ of the study population, which suggests a decrease in EBV infection in Saudi Arabia.

Apart from other risk factors, HPV is also one of the emerging causes of virus-related HNC incidence, particularly in HNSCC, worldwide ${ }^{22,23}$. In comparison to EBV, HPV is considered more commonly responsible for causing HNSCC in western countries. This might be attributed to the changing sexual behaviours during recent decades ${ }^{24}$. Various HNC malignancies have been associated with HPV such as cancer at the pharynges, the base of the tongue, and tonsils (oropharynx area). According to a study by Kreimer et al. ${ }^{25}$, about $35.6 \%$ of oropharyngeal, $23.5 \%$ of oral and $24 \%$ of 
laryngeal cancers were associated with HPV. Due to cultural and religious boundaries in Saudi Arabia, the real impact of HPV on the HNCs has not been investigated to a full extent. Only a few reported studies are available that have reported a link between HNC and HPV 2 .

The most important prognostic factor for HNC patients is the clinical staging of the tumor. Accurate mapping of the tumor is critical before commencing therapy. The tumor is categorised according to TNM staging, which assigns tumors as local (T), regional nodal (N) or distant (M). Tumors of the head and neck can further be classified from stage 0 to IV, based on the size of the tumor and extended presence from their site of initiation ${ }^{26}$. In this study, the majority of HNC patients belonged to TNM stages III (15.1\%) and IV (64.6\%). A single modality treatment with a 5 -year survival rate of $60-90 \%$ is required for early stages (I and II), while advanced stages (III and IV) require a combined modality and have a 5 -year survival rate of $<35 \%{ }^{27}$. According to a study, most (66.6\%) of the cases of HNC are reported at an advanced stage (Stages III and IV) in Saudi Arabia ${ }^{5}$. In the present study as well, $79.7 \%$ of the patients had advanced stage HNC, which might be due to the poor awareness of the disease and limited hospital resources.

Various modalities such as recurrent/metastatic disease chemotherapy, biological treatment, radiation therapy, immunotherapy and extensive surgical resection of either primary tumor or lymph nodes are available as standard options in the treatment of HNC that can render a median survival of $6-12$ months and a $20-40 \%$ rate of 1 -year survival $^{28}$. However, the pattern of a major failure in the treatment of HNC is still represented by the local or regional recurrences. The problem is more challenging in patients with advanced stages. Maklad et al. ${ }^{29}$ reported an overall failure of treatment in 21 out of 68 Saudi patients with nasopharyngeal cancer. Out of 21, seven were locoregional, 3 were regional with DM, and 11 had DM. Similarly, in another study by Al-Herabi, out of $44 \mathrm{HNC}$ patients, $2(5 \%)$ patients died perioperatively, and $2(5 \%)$ patients had a locoregional recurrence $^{30}$. In our study, there were $24.3 \%$ treatment failures, and about $62.5 \%$ of patients were recorded with metastasis.

An improvement in the management of HNC can be expected with an early referral of the suspected individual to the specialty centres, which can enhance the survival outcome. The treatment of HNC varies according to the primary site, tumor stage, patient treatment preference, and practitioner's expertise ${ }^{31}$. The management of HNC requires a multifaceted approach that involves oncologists with expertise in medicine, radiology and surgery, dentists with expertise in $\mathrm{HNC}$, radiologists, speech therapists, social volunteers (workers), psychologists, and plastic and/or reconstructive surgeons ${ }^{32}$.

In our study, the treatment failures were recorded as local as well as distant. The metastasis was also observed in patients during their subsequent visits. The nasopharyngeal and oral cavity cancers had the highest number of regional failures as well as distant failures. Our study is in accordance with the study by Leeman et al. ${ }^{33}$ that showed a majority of regional failures for oral cavity cancer.

The lack of knowledge about the disease in our patients is evident, which further hampers clinical understanding and delays timely diagnosis and treatment. Thus, the development of proper treatment strategies for this subgroup of patients as well as further research into more effective diagnosis and treatment are warranted.

\section{Strengths and limitations}

We consider our findings significant, as the results confirmed the pattern of HNC in the Saudi population in terms of its subtype, histological prevalence, and failures of treatment. The findings of this study are expected to guide the strategies to be adopted towards improvement in obtaining local and/ or regional control or reducing the development of DM. However, the study has limitations in terms of being a singlecentre retrospective study and having a relatively small sample size. Owing to this, complete information regarding etiological factors and $\mathrm{N}$ and $\mathrm{M}$ stages separately was not available.

\section{CONCLUSIONS}

The study highlights the increased use of tobacco in the region, which needs to be monitored by conducting epidemiological surveys and controlled by spreading awareness and educating people. There is a need to improve diagnosis and treatment modalities in the region. Moreover, regular training of healthcare professionals is also essential for early diagnosis. Patient factors, such as willingness to seek treatment, are also significant. These factors may have influenced the relatively low percentage of survival rate. However, a multicenter study with an extensive series of cases is required to confirm the present findings.

\section{REFERENCES}

1. Sharma JD, Baishya N, Kataki AC, Kalita CR, Das AK, Rahman T. Head and neck squamous cell carcinoma in young adults: A hospital-based study. Indian J Med Paediatr Oncol. 2019;40(5):18-22. doi:10.4103/ijmpo.ijmpo_252_17

2. Alsbeih G, Al-Harbi N, Bin Judia S, Al-Qahtani W, Khoja H, El-Sebaie M, Tulbah A. Prevalence of Human Papillomavirus (HPV) Infection and the Association with Survival in Saudi Patients with Head and Neck Squamous Cell Carcinoma. Cancers. 2019;11(6):820. doi:10.3390/cancers11060820

3. Elasbali AM, Ahmed HG. A Review on the Etiology of Oral Cancer in Saudi Arabia. Int J Med Res Health Sci. 2018;7(6):161-170. https://www.ijmrhs.com/medicalresearch/a-review-on-the-etiology-of-oral-cancer-in-saudiarabia.pdf. Accessed January 10, 2020.

4. Kobayashi K, Hisamatsu K, Suzui N, Hara A, Tomita H, Miyazaki T. A Review of HPV-Related Head and Neck Cancer. J Clin Med. 2018;7(9):241. doi:10.3390/jcm7090241 
5. Alhazzazi TY, Alghamdi FT. Head and Neck Cancer in Saudi Arabia: a Systematic Review. Asian Pac J Cancer Prev. 2016;17(8):4043-4048. PMID:27644659.

6. Al-Wassia R, Abusanad A, Awad N, Marzouki H, Alkhayyat S, Al-Khatib T, Constantinescu C. Outcomes of Saudi Arabian patients with nasopharyngeal cancer treated with primarily neoadjuvant chemotherapy followed by concurrent chemoradiotherapy. J Glob Oncol. 2016;2(3):123-128. doi:10.1200/jgo.2015.001743

7. Tandon P, Pathak VP, Zaheer A, Chatterjee A, Walford N. Cancer in the Gizan Province of Saudi Arabia: an eleven year study. Ann Saudi Med. 2015;15(1):14-20. doi:10.5144/0256-4947.1995.14

8. Cohen EE, LaMonte SJ, Erb NL, Beckman KL, Sadeghi N, Hutcheson KA, Pratt-Chapman ML. American Cancer Society Head and Neck Cancer Survivorship Care Guideline. CA Cancer J Clin. 2016;66(3):203-239. doi:10.3322/caac.21343

9. Kim DH, Kim WT, Lee JH, Ki YK, Nam JH, Lee BJ, Kim DW. Analysis of the Prognostic Factors for Distant Metastasis after Induction Chemotherapy Followed by Concurrent Chemoradiotherapy for Head and Neck Cancer. Cancer Res Treat. 2015;47(1):46-54. doi:10.4143/crt.2013.212

10. Pulte D, Brenner H. Changes in survival in head and neck cancers in the late 20th and early 21st century: a period analysis. Oncologist. 2010;15(9):994-1001. doi:10.1634/theoncologist.2009-0289

11.El Awa F. Tobacco control in the Eastern Mediterranean Region: overview and way forward. East Mediterr Health J. 2008;14(Suppl):S123-S131. PMID:19205612.

12. Lydiatt WM, Patel SG, O'Sullivan B, Brandwein MS, Ridge JA, Migliacci JC, Shah JP. Head and neck cancers-major changes in the American Joint Committee on cancer eighth edition cancer staging manual. CA Cancer J Clin. 2017;67(2):122137. doi:10.3322/caac.21389

13. Reichart PA, Way TH. Oral cancer and pre-cancer in Myanmar: a short review. J Oral Pathol Med. 2006;35(4):193196. doi:10.1111/j.1600-0714.2006.00390.x

14. Al-Zahrani O, Eldali A, Al-Shahri MZ. Prevalence and severity of pain in cancer patients in an outpatient palliative care setting in Saudi Arabia. Qatar Med J. 2014;2014(1):38-45. doi:10.5339/qmj.2014.6

15. Farrag A, Shaukat A, Ali M, Kandil M. Clinico-Pathological Features and Outcome of Treatment of Saudi Patients with Oral Cavity Cancer. Head Neck Cancer Res. 2018;3(1). doi:10.21767/2572-2107.100022

16. Cruz-Gregorio A, Martínez-Ramírez I, Pedraza-Chaverri J, Lizano M. Reprogramming of energy metabolism in response to radiotherapy in head and neck squamous cell carcinoma. Cancers. 2019;11(2):182. doi:10.3390/cancers11020182

17. Chang ET, Adami HO. The enigmatic epidemiology of nasopharyngeal carcinoma. Cancer Epidemiol Biomarkers Prev. 2006;15(10):1765-1777. doi:10.1158/1055-9965.EPI-06-0353

18. Hesham A, Syed KB, Jamal BT, et al. Incidence, clinical presentation, and demographic factors associated with oral cancer patients in the southern region of Saudi Arabia: A 10- year retrospective study. Journal of International Oral Health. 2019;9(3):105-109. doi:10.4103/jioh.jioh_58_17

19. Raab-Traub N. Epstein-Barr virus in the pathogenesis of NPC. Semin Cancer Biol. 2002;12(6):431-441. doi:10.1016/s1044579x0200086x

20.Young LS, Dawson CW. Epstein-Barr virus and nasopharyngeal carcinoma. Chin J Cancer. 2014;33(12):581590. doi:10.5732/cjc.014.10197

21. Nasrin N, Taiba K, Hannan N, Hannan M, al-Sedairy S. A molecular study of EBV DNA and p53 mutations in nasopharyngeal carcinoma of Saudi Arab patients. Cancer Lett. 1994;82(2):189-198. doi:10.1016/0304-3835(94)90011-6

22. Boscolo-Rizzo P, Del Mistro A, Bussu F, Lupato V, Baboci L, Almadori G, DA Mosto MC, Paludetti G. New insights into human papillomavirus-associated head and neck squamous cell carcinoma. Acta Otorhinolaryngol Ital. 2013;33(2):77-87. PMID:23853396.

23. Gillison ML, Chaturvedi AK, Anderson WF, Fakhry C. Epidemiology of Human Papillomavirus-Positive Head and Neck Squamous Cell Carcinoma. J Clin Oncol. 2015;33(29):3235-42. doi:10.1200/JC0.2015.61.6995

24.Azimi S, Mortazavi H, Tennant M, Kruger E, Rezaei B, Taheri JB, Tarahhomi MR. Pattern of the head and the neck cancer in two geographically and socioeconomically different countries. J Orofac Sci. 2017;9(1):43-47. doi:10.4103/0975-8844.207943

25. Kreimer AR, Clifford GM, Boyle P, Franceschi S. Human papillomavirus types in head and neck squamous cell carcinomas worldwide: a systematic review. Cancer Epidemiol Biomarkers Prev. 2005;14(2):467-475. doi:10.1158/1055-9965.EPI-04-0551

26. Worsham MJ. Identifying the risk factors for late-stage head and neck cancer. Expert Review of Anticancer Therapy. 2011;11(9):1321-1325. doi:10.1586/era.11.135

27. Gil Z, Fliss DM. Contemporary management of head and neck cancers. Isr Med Assoc J. 2009;11(5):296-300. PMID:19637508.

28. Cognetti DM, Weber RS, Lai SY. Head and neck cancer: an evolving treatment paradigm. Cancer. 2008;113(7 Suppl):1911-1932. doi:10.1002/cncr.23654

29. Maklad AM, Bayoumi Y, Senosy Hassan MA, et al. Patterns of failure and survival in patients with nasopharyngeal carcinoma treated with intensity-modulated radiation therapy in Saudi Arabia. Onco Targets Ther. 2016;9:65616567. doi:10.2147/OTT.S95457

30. Al-Herabi AZ. Head and neck oncology experience in Makkah, Saudi Arabia. Saudi Med J. 2009;30(10):1316-1322. PMID:19838441.

31. Gilyoma JM, Rambau PF, Masalu N, Kayange NM, Chalya PL. Head and neck cancers: a clinico-pathological profile and management challenges in a resource-limited setting. BMC Res Notes. 2015;8:772. doi:10.1186/s13104-015-1773-9

32. Sanderson R, Wei WI, Ironside J. Clinical reviewSquamous cell carcinomas of the head and neck Commentary: Head and neck carcinomas in the developing world. BMJ. 
2002;325(7368):822-827.

33. Leeman JE, Lee N, Riaz N. Patterns of failure in head and neck cancer patients treated with intensity modulated radiation therapy: editorial response. Translational Cancer Research. 2017;6(S7):S1299-S1300. doi:10.21037/tcr.2017.10.04

CONFLICTS OF INTEREST

The authors have completed and submitted the ICMJE Form for Disclosure of Potential Conflicts of Interest and none was reported.
PROVENANCE AND PEER REVIEW

Not commissioned; externally peer reviewed.

FUNDING

There was no source of funding for this research. 